\title{
O Programa Saúde da Família no Brasil: análise do acesso à atenção básica
}

\author{
The Family Health Program in Brazil: analysis of access to basic care \\ El Programa Salud de la Familia en Brasil: analísis del acceso a la atención basica
}

\author{
Maria Fátima de Sousa' \\ 'Universidade de Brasília, Núcleo de Estudos em Saúde Pública. Brasília, DF
}

Submissão: 01/09/2007

Aprovação: 01/12/2007

\section{RESUMO}

Este artigo apresenta dados de uma pesQuisa sobre a implantação do Programa de Saúde da Família-PSF, realizada nos 12 municípios pioneiros, no Que se refere ao acesso aos serviços básicos de saúde. Fundamentou-se, no contexto das ciências sociais, no método da hermenêutica-dialética. Foram utilizados roteiros de entrevista e grupos focais para coleta de dados. Evidenciou o estudo, no Que se refere ao acesso aos serviços básicos de saúde, o PSF vem contribuindo para a diminuição das inieüidades. Enfrenta, no entanto, uma série de desafios de ordem social, político, econômico institucional e cultural, materializados nas deficiências de coordenação, gestão, financiamento, e, sobretudo nas praticas médico assistencial, fortemente presentes no cotidiano do trabalho das eQuipes do PSF.

Descritores: Programa Saúde da Família; Atenção primária à saúde; Acesso aos seviços de saúde.

\section{ABSTRACT}

This article shows a research about the implantation of the Family Helath Program, carried through 12 pioneer cities, related to the access to the basic health services. It was based in the context of social sciences, in the method of the hermeneutics-dialectics. Scripts of interview and focal groups for data collection were used. The study highlights regarding to the primary care accessibility to health sercives, that the PSF has contributed on the reduction of inequalities. However, it faces a sort of social, political, institutional, economic and cultural challenges, that are materialized in the deficiencies of coordination, management, financing, and mainly in medical practical assistance, strongly present in the daily professional practice of the PSF's teams.

Descriptors: Family Health Program; Primary health care; Health services acessibility.

\section{RESUMEN}

Este artículo presenta datos de una investigación acerca de la implantación del Programa Salud de la Familia (PSF), realizada en las 12 municipalidades pioneras del programa, sobre el acceso a los servicios básicos de la salud. Fue basado en el contexto de ciencias sociales, en el método de la hermenéutica-dialectica. Los discursos de la entrevista y de los grupos focales para la recogida de datos fueron utilizadas. El estudio evidenció, cuanto al acceso a los servicios básicos de la salud, Que el PSF hay contribuydo para la reducción de las diferencias. Sin embargo, enfrenta una serie de desafíos de orden social, política, institucional, cultural, económica, Que son materializados en las deficiencias de la coordinación, de la gerencia, del financiamiento, y sobre todo en las practicas medico asistenciales, fuertemente presentes en el cotidiano del trabajo de los esQuipos del PSF.

Descriptores: Programa Salud de la Familia; Atención primaria de salud; Accesibilidad a los servicios de salud. 


\section{INTRODUÇÃO}

Pode-se afirmar Que o SUS, garantido pela Constituição Federal e leis orgânicas Lei N. ${ }^{\circ} 8.080$, de 19 de setembro de 1990, pela Lei $N^{\circ} 8.142$, de 28 de dezembro de 1990, com seus princípios doutrinados e preceitos constitucionais de universalidade (acesso da população a Qual Quer serviço de saúde); eQüidade (acesso em igualdade de condições aos diferentes níveis de complexidade do sistema) e integralidade (ações integrais e não mais fragmentadas) representam um grande avanço no projeto de Reforma Sanitária Brasileira. Entretanto, tem sido insuficiente para promover as transformações necessárias para a construção de um novo modelo de atenção à saúde integral e familiar.

Sabe-se que o Brasil já viveu ao longo das últimas décadas uma série de tentativas de mudar, na expressão de Teixeira ${ }^{(1)}$, a forma e o conteúdo de prestar atenção à saúde. No entanto, todas as iniciativas, por melhor Que tenha sido a origem de suas propostas em termos de base filosófica, princípios e diretrizes, não foram suficientes para alterar as bases Que sustentam o modelo médico assistencial predominante no país.

Essas bases não sofrerão alterações porQue traziam em seus cernes elementos parciais dos problemas complexos Que se enfrentam na construção de um novo modelo. Tal complexidade vem sendo retratada ao longo das décadas por autores diversos, entre eles Arouca ${ }^{(2)}$, Donangelo ${ }^{(3)}$, Paim ${ }^{(4-6)}$, Garcia ${ }^{(7)}$, MendesGonçalves $^{(8)}$ e Teixeira ${ }^{(1,9,10)}$. Eles nos alertam para a necessidade de compreendermos os fatores e as dinâmicas sociais, econômica, política e cultural Que determinam a hegemonia de modelo sanitário, centrado nos hospitais, nas subespecialidades, nas ações de programas verticais e nas práticas médicas baseadas na doença e nos aparatos medicamentosos.

É nesse cenário dos velhos e novos problemas, Que nasceu o Programa Saúde da Família (PSF,) em março de 1994, como uma estratégia política para promover a organização das ações de atenção básica à saúde nos sistemas municipais de saúde; está inserido em um contexto de decisão política e institucional de fortalecimento da Atenção Básica no âmbito do Sistema Único de Saúde e se fundamenta em uma nova ética social e cultural, com vistas à promoção da saúde e da Qualidade de vida dos indivíduos, famílias e comunidades. E seus princípios organizativos revelam-se nos seguintes objetivos: a) prestar, na unidade de saúde e no domicílio, assistência integral, resolutiva, contínua, com resolubilidade e boa Qualidade às necessidades de saúde da população adscrita; b) intervir sobre os fatores de risco aos Quais a população está exposta; c) eleger a família e o seu espaço social como núcleo básico de abordagem no atendimento à saúde; d) humanizar as práticas de saúde através do estabelecimento de um vínculo entre os profissionais de saúde e a população; e) proporcionar o estabelecimento de parcerias através do desenvolvimento de ações intersetoriais; f) contribuir para a democratização do conhecimento do processo saúde/doença, da organização dos serviços e da produção social da saúde; g) fazer com Que a saúde seja reconhecida como um direito de cidadania e, portanto, expressão da Qualidade de vida; e (viii) estimular a organização da comunidade para o efetivo exercício social $^{(11)}$.

Passada mais de uma década de funcionamento do PSF no Brasil, resolvemos estudar sua implantação nos 12 municípios pioneiros, com leitura da realidade, caso a caso de forma a compreender melhor a(s) resposta(s) à Questão central da pesquisa, Que foi, $\mathbf{O}$ PSF é uma estratégia de superação da desigualdade na saúde?

\section{MÉTODO}

Para tanto, o método do estudo fundamentou-se, no contexto das ciências sociais, na hermenêutica-dialética; uma pesQuisa Quantitativa, cujos métodos e técnicas consistiram em coletar, analisar, compreender e interpretar os discursos dos sujeitos coletivos selecionados entre os gestores do SUS. Os discursos dos sujeitos foram organizados e analisados a partir da técnica de Discurso do Sujeito Coletivo - DSC de Lèfevre e Lèfevre ${ }^{(12)}$.

O trabalho tomou para análise do acesso aos serviços básicos de saúde, as seguintes dimensões: a política, a técnica-financeira e a simbólica. A dimensão política considera as motivações Que levaram os sujeitos político-técnicos à decisão de implantar o PSF, nos três níveis de governo (federal, estadual e municipal) e ao implantarem se tomaram em conta a possibilidade de reduzir as desigualdades de acesso aos serviços básicos de Saúde. A dimensão técnica-financeira se refere à expansão da cobertura (cobertura geográfica) verificando os critérios e prioridades de exclusão e/ou inclusão na decisão dos sujeitos político-técnicos, Quando da escolha de áreas nas Quais seria implantado, e se a mesma favorece ou não a redução das desigualdades de acesso. Ainda nessa dimensão foram considerados os elementos de governança institucional do PSF verificando as capacidades dos governos em implantar e ou implementar o Saúde da Família nas três instâncias gestoras do SUS. Finalmente, a dimensão simbólica trata da percepção do acesso pelos usuários no Que diz respeito à forma de inter-relação estabelecida entre esses sujeitos, os gestores e as equipes do PSF; permeada pelo vínculo e pela coresponsabilidade, num exercício permanente de geração de oportunidades e capacidades entre os sujeitos nos processos de cuidar da saúde-doença-morte como expressões de respeito, autonomia e participação.

\section{RESULTADOS E DISCUSSÃO}

\section{A força da Realidade: Discursos dos Sujeitos Coletivos}

$\mathrm{Na}$ dimensão política, perguntamos aos gestores do SUS, das três instâncias de governo, Quais as motivações Que os levaram a implantar o PSF. E ao implantarem se tomaram em conta a possibilidade de reduzir as desigualdades de acesso aos serviços básicos de Saúde. Os discursos analisados demonstram Que as motivações se apresentam, segundo os lugares, responsabilidades e competências de cada nível de gestão do SUS. Portanto, as falas desses sujeitos são conseqüências dos espaços de poder por eles ocupados. Os discursos dos Gestores Nacionais evidenciam Que suas motivações para induzir a implantação do PSF estavam diretamente vinculadas ao desejo de Que essa estratégia pudesse contribuir para a organização do SUS e reordenamento do sistema; enquanto que os depoimentos dos Secretários de Estados da Saúde e dos Coordenadores da Atenção Básica/PSF, (Gestores Estaduais) demonstraram Que suas motivações decorreram da vontade de ver no PSF uma estratégia com o potencial de mudança 
do modelo assistencial. Já as falas dos respondentes no âmbito dos sistemas municipais de saúde (Gestores Municipais Secretários e Coordenação da Atenção Básica - PSF) apontaram para a necessidade de Que o PSF pudesse de fato re-organizar a Atenção Básica. Cabe-nos destacar ainda que os Estados Que responderam o roteiro de entrevista foram: no Nordeste - Bahia, Ceará, Paraíba, Pernambuco e Sergipe; na região Sudeste - São Paulo e Minas Gerais; no Sul - Santa Catarina; no Norte - Roraima e Manaus; na região Centro-oeste Goiás e Mato Grosso.

Essas foram as idéias centrais expressas nos discursos desses sujeitos Que têm responsabilidades e competências diferenciadas nas intervenções dos processos de expansão e Qualificação do PSF no país. Nelas fica claro Que suas motivações para implantação e/ou implementação dessa estratégia, em alguma medida, levou em consideração a possibilidade de reduzir as desigualdades de acesso aos serviços básicos de saúde.

$\mathrm{Na}$ dimensão técnica-financeira, os discursos dos sujeitos coletivos não podem ser tomados de forma isolada, Quando se trata de compreender a categoria analítica "expansão da cobertura do PSF no Brasil” (distribuição geográfica); e sim triangulados a outras fontes de dados, a exemplo das pesquisas realizadas para identificar a cobertura e ou distribuição das equipes do PSF no Brasil, entre elas Evolução do processo de implantação do PSF no Brasil - 1998-2001; Saúde da Família no Brasil - uma análise de indicadores selecionados - 1998-2004 e Avaliação da implementação do Programa de Saúde da Família em dez grandes centros urbanos, 2005, todas realizadas pelo Ministério da Saúde/ SAS/DAB. Apesar da rieueza dos dados, observam-se lacunas de acesso às ações e serviços básicos de saúde. Estas ocorrem desde a origem da indução pelo Ministério da Saúde à implantação dessa estratégia. Naquela época somente 12 estados da federação e 12 municípios aderiram a esta iniciativa, com apenas 328 equipes em funcionamento. Atualmente 5.131 municípios brasileiros contam com 27.454 equipes do PSF, dando a oportunidade a 87.916,762 milhões de pessoas de serem cuidadas o mais próximo possível dos seus lugares de moradia ${ }^{(13)}$. No entanto, essa cobertura ainda expressa a distribuição desigual das equipes do PSF no conjunto das cidades brasileiras.

É esse contexto Que explica e fundamenta, por um lado, a pronta adesão dos municípios de pequeno porte - entre 10 e 20 mil habitantes; e médio porte - de 20 a 50 mil habitantes, por estes não disporem de uma rede de serviços previamente estruturada, com baixa ou nenhuma capacidade instalada, vindo a fazê-la com a inserção das equipes do PSF. Por outro lado, as capitais e os municípios acima de 100 mil habitantes, onde reside mais de $50 \%$ da população brasileira, continuam com baixas coberturas, principalmente entre as cidades Que concentram o maior número de pessoas e entre os principais estados e regiões do país.

Além disso, pode-se observar nessa pesQuisa a disputa políticopartidária entre estados e municípios, Quando suas gestões estão sob o comando de partidos políticos contrários; nesses cenários, as resistências e oposição corporativa e ideológica se ampliam, dificultando com isso a expansão do PSF. Adicionam-se a esse Quadro outros fatores Que contribuem para a baixa cobertura nas capitais, nos grandes centros metropolitanos, a exemplo de hesitações e dúvidas dos gestores municipais Quanto às evidências e benefícios de um sistema de saúde orientado pelas ações e serviços básicos de saúde; a pressão política, social, e por vezes da imprensa, por demandas pontuais de cunho assistencial; a existência de uma rede física instalada centralizada nos hospitais e ambulatórios de subespecialidades; a concentração de recursos clínicos de alta e média complexidade; o acúmulo de demandas vindas de outros municípios Que não dispõem de recursos, sobretudo de apoio diagnóstico e terapêutico, concentrados nas capitais e ou regiões metropolitanas; os municípios não atendem apenas às demandas de sua população; a insegurança em transitar de uma rede tradicional para uma rede focada na lógica organizativa do PSF, com a imposição de novos problemas de infra-estrutura e recursos humanos; e as demandas da população por especialistas.

Vários autores assinalam como fato observado a existência de boas práticas por parte das equipes do PSF, sobretudo nos municípios de pequeno porte ${ }^{(1,14)}$. Porém, muitas vezes estas práticas apresentam-se limitadas para a oferta de atenção integral face à ausência de uma rede regionalizada de referência e contra-referência de serviços assistenciais. Logo, a obtenção de boas práticas não altera substantivamente a lógica organizativa dos serviços e sistemas locais de saúde, predominando com isto o modelo clássico de assistência a doenças em suas demandas espontâneas, sustentadas no tripé do médico Que realiza consultas com apoio diagnóstico, equipamentos e medicamentos.

Para análise dessa situação, Teixeira ${ }^{(1)}$ nos instiga sobre a pertinência de expandir essa estratégia para os municípios acima de 100.000 habitantes da mesma forma como vem sendo implementada nos demais. Nesses novos espaços-territórios o PSF passa a concorrer fortemente com as práticas assistenciais médicocurativas, o Que provoca tensão e resistência de atores envolvidos na manutenção do modelo hegemônico.

Essas tensões e resistências também se expressam de uma outra maneira nos 12 municípios pesquisados, sobretudo, no momento de definição dos critérios e prioridades de exclusão e/ou inclusão das equipes do PSF a serem implantadas, ainda que levando em consideração a redução das desigualdades de acesso aos serviços básicos de saúde, mas a concretude desse acesso se depara com problemas estruturais Que vão desde a fragilidade financeira, em função da baixa capacidade de arrecadação tributária, passando pelo conflito da lei de responsabilidade fiscal que limita a contratação de novos profissionais, pelas debilidades na infra-estrutura da rede física instalada, e, culminado nas demandas insistentes por atendimento a doença e seu aparato médico-assistencial.

Uma outra categoria analítica - incentivos financeiros, ainda na dimensão técnica-financeira - no Que se refere aos investimentos para expansão com Qualidade do PSF, foram verificadas nos 12 municípios pesquisados três situações limitantes: a primeira se apresenta na constatação das desigualdades no financiamento entre os níveis de atenção do sistema. Os números apontam os desequilíbrios, ainda fortes, entre os recursos direcionados para a média e alta complexidades, situação predominante e induzida nas formulações das políticas do Ministério da Saúde. A segunda se encontra na ausência e ou no baixo co-financiamento pelas Secretarias de Estado da Saúde, entes federados que deveriam apoiar os municípios na co-responsabilidade dos processos reorganizativos da Atenção Básica. E a terceira limitação, conseQüência das anteriores, se acentua Quando os municípios dos grandes centros urbanos (capitais, regiões metropolitanas e acima 
de 100 mil habitantes) são tratados de formas iguais, Quando suas realidades impõem a definição de uma política de co-financiamento dos três níveis de governo diferenciada da forma como vêm sendo tratados os municípios de pequeno porte.

O objetivo é que uma nova política atenda aos problemas típicos desses espaços/territórios onde reside mais de $50 \%$ da população brasileira. Nos discursos dos sujeitos coletivos é vista a ressalva Que os gestores municipais não desejam privilégios, apenas reivindicam o direito de serem tratados em suas diferenças, de forma a superar as desigualdades.

Encontrar respostas para essas e outras limitações no tocante à Questão do financiamento da Atenção Básica/PSF, nos impõe novos desafios, principalmente com relação à prática co-operativa e complementar construída entre os gestores do SUS. Tudo isto lembra a necessidade de definir mecanismos de financiamento que contribuam para a redução das desigualdades regionais e para uma melhor proporcionalidade entre os três níveis de atenção do SUS com primazia para a expansão dos recursos alocados ao $\operatorname{PSF}^{(15)}$.

Neste caso, as esferas de governo - federal, estadual e municipal - devem estabelecer pactos de co-responsabilidade no financiamento das ações e dos serviços de saúde, provendo o direito à saúde de forma responsável e solidária. Com isto, a estratégia Saúde da Família - eixo prioritário de investimento da Atenção Básica - deve contar com financiamento específico, proveniente da responsabilidade constitucional, também, do governo estadual.

$\mathrm{Na}$ dimensão simbólica tratamos da percepção do acesso pelos usuários aos serviços básicos de saúde. Nessa categoria analítica, verificou-se nas afirmativas dos discursos dos sujeitos coletivos (usuários) do PSF, Que a percepção do acesso aos serviços básicos de saúde resume-se à noção de doença e de suas formas medicamentosas de tratá-las. Com isso, é importante ter em conta Que essas noções, crenças vêm sendo culturalmente construídas de geração a geração, conscientemente através do grupo familiar e outras instituições, e, inconscientemente, através de mecanismos de reprodução do modelo médico assistencial, Que em certa medida é promovida pelo Aparelho de Estado. Entretanto, compreende-se em alguns discursos desses sujeitos a inversão dessa cultura Quando as eQuipes do PSF assumem a função social, com o protagonismo dos Agentes Comunitários de Saúde, de contribuir na criação de capacidades comunitárias na problematização do estado de saúdedoença-cuidado, tendo como âncora a consciência sanitária.

\section{Limites do Acesso aos Serviços Básicos de Saúde:} Velhos Desafios, Novos Caminhos

O acesso aos serviços básicos de saúde no âmbito do SUS, segundo nosso estudo apresenta-se limitado em três aspectos principais: o primeiro pela baixa capacidade de integração entre as eQuipes da atenção básica (unidades tradicionais), as equipes do PSF, e entre estas, naquilo Que se refere ao planejamento e à programação das unidades básicas, tendo como referência o Sistema de Informação da Atenção Básica - SIAB; este vem sendo utilizado de forma limitada e burocrática, comprometendo, portanto, a prática da institucionalização de instrumentos e mecanismos de monitoramento e avaliação das ações e serviços básicos de saúde. A conseQüência desse Quadro é a reprodução e/ou manutenção do modelo centrado no médico e em tecnologias de prevenção, tratamento e cura das doenças, sendo visível a incipiência das práticas de trabalho em equipe, onde ficam claros para os profissionais as responsabilidades de cada um e o compromisso de todos com a saúde das famílias e comunidades.

O segundo limite encontra-se expresso na desintegração entre as eQuipes da atenção básica; nesse caso, a pesquisa restringe-se ao PSF e aos demais pontos do sistema (média e alta complexidade). Com isso tornam-se vulneráveis a continuidade e a integralidade da atenção, uma vez Que em Quase todos os 12 municípios não existe uma política de referência e contra-referência, ficando esta responsabilidade para os profissionais mais "engajados" com os propósitos do PSF, no plano da informalidade e/ ou amizade entre pares, sobretudo entre os enfermeiros. Poucos são os municípios Que começam a estruturar suas centrais de regulação, com vistas a atender as demandas das equipes do PSF nas ações e serviços de consultas especializadas e/ou apoio diagnóstico (exames). A esse respeito deve-se ressaltar Que os discursos dos sujeitos coletivos (equipes do PSF) registram Que os ACS são os principais elos entre as unidades básicas de saúde e as comunidades, cabendo a estes a responsabilidade de articular o acesso a consultas, exames e/ou medicamentos, nas unidades básicas e de especialidades. Vários foram os municípios em Que verificamos ser o ACS o articulador também das consultas juntos aos médicos do PSF, estes cumprindo apenas a "tarefa" de realizar as consultas programadas e ou solicitadas pelos Agentes. Os demais membros da ESF vêem o ACS como porta-vozes das ações programáticas do ciclo de vida, onde as unidades permanecem realizando ações dirigidas aos programas de forma isolada e verticalizada.

O terceiro limite é verificado na baixa capacidade das equipes da atenção básica/PSF em elaborarem planos, programas, projetos e/ou ações contínuas Que potencializem suas relações e integrações com outros setores do governo e da sociedade. Estas fragilidades são perceptíveis nas falas dos sujeitos pesquisados Quando afirmam compreender Que as ações de promoção extrapolam o setor, e por estas razões não dispõem de tempo e "governabilidade" para investir nessas iniciativas, além de serem pressionados pelas demandas de ordem assistencial e curativa. Reconhecem Que as ações de promoção e educação em saúde dariam sustentação às suas atividades, na medida em Que estas valorizam os determinantes gerais do complexo saúde-doença-cuidado. Reconhecem, sobretudo, Que para redirecionar suas práticas rumo à promoção da saúde, é necessário integrar as políticas públicas governamentais e não governamentais, visando modificar as condições de vida e saúde, indo, portanto para além de ações educativas individuais e de mudanças de estilos de vida.

Apesar disso, metades dos municípios pesquisados apresentamse preocupados e "conscientes" de Que o acesso aos serviços básicos de saúde não deve ficar restrito aos atributos da adeQuação das unidades básicas, do uso de serviços e da assistência à doença. Todavia, sinalizam estes municípios que os gestores do SUS devem se ocupar com estes atributos, sem esquecer de adicionar a eles a Questão das inter-relações entre gestores, equipes e comunidades na adeQuação de tecnologias humanizadas no ato de cuidar da saúde das famílias.

Os velhos desafios e novos caminhos a superação das desigualdades na saúde foram elementos de análises e interpretações dos dados do estudo, na medida em Que indagamos ser o PSF uma estratégia de superação das iniQüidades. Os achados 
nos fazem compreender: Primeiro no Que se refere às desigualdades na saúde, o PSF isoladamente não responde a essa responsabilidade social, uma vez Que esta Questão extrapola o âmbito das políticas públicas universais desse setor. A saúde em si mesma não resolve as desigualdades como demonstra as brechas existentes, ainda Que pequenas, em paises sociais democráticas e/ou em estados que adotam políticas de bem-estar social, a exemplo da Inglaterra, Suécia, Noruega, entre outros. Portanto, a superação das desigualdades na saúde estar intrinsecamente ligada ao determinante do desenvolvimento político, econômico, social e ambiental.

No sentido limitador, a situação da saúde se depara no caso brasileiro com um sistema de proteção social, Que apesar dos seus avanços, continua incompleto, frágil e com baixa capacidade de afetar positiva e significativamente os indicadores de desigualdade social e de saúde. A triangulação dos dados informa Que este sistema trouxe para o novo século as duas principais marcas da situação social Que experimentou praticamente durante todo o século passado: uma das mais desiguais estruturas sociais entre os países de médio e alto desenvolvimento econômico e um modelo paradigmático de gestão do Aparelho de Estado centrado em estruturas clientelistas de manutenção do poder patrimonialista. Com efeito, ainda é ampla a agenda de desejo de mudanças a ser percorrida pelo sistema em busca da superação das desigualdades em saúde, em um Estado e sociedade orientados pela democracia e pela justiça social. Segundo, no Que diz respeito à desigualdade de acesso aos serviços básicos de saúde, o PSF também não pode responder de forma isolada por esta "tarefa", uma vez que ainda não se constitui em uma política universal, com a clara definição econômica, social, cultural e ideológica dessa finalidade e/ou objetivo. Por isso a vulnerabilidade interna da atenção básica, desta em relação ao sistema e desse com os outros setores da sociedade, confirma as limitações acima citadas. Terceiro - os discursos apresentados nesta pesQuisa assinalam claramente Que, na visão dos gestores, o PSF é uma estratégia política com um contribuinte potencial para diminuição das iniQüidades na atenção básica, ampliando o acesso nesse nível da atenção, mas não supera as desigualdades na saúde, porque esta é uma das Questões amplas e complexas Que exige alterar os fatores determinantes políticos, sócio-culturais, econômicos e ambientais.

\section{CONSIDERAÇÕES FINAIS}

Por fim, o Que podemos afirmar, diante das produções já existentes nos 12 municípios pesquisados e nas referências tomadas pelas Secretarias de Estados da Saúde e pelo Ministério da Saúde, é que o PSF vem contribuindo na ampliação do acesso aos serviços básicos de saúde, mas, é preciso enfrentar os desafios contemporâneos Que essa estratégia apresentam no cotidiano das praticas de suas equipes, e mais que os gestores do SUS devem tomem para si esta responsabilidade, no sentido de imprimir as reformas do setor saúde, e estas orientadas pelos valores, princípios e características organizativos da atenção básica, tendo no PSF a estratégia em primazia.

Essa construção não pode prescindir de uma outra Questão desafiadora, ou seja, a de produzir tecnologias de gestão aplicáveis aos processos de monitoramento e avaliação, colocando no centro da discussão os seguintes aspectos: a Qualidade da expansão das equipes do PSF, a utilização do sistema de informação como ferramenta gerencial Que contemple a complexidade da organização da atenção e apresente indicadores adequados e oportunos para acompanhamento dos efeitos e impactos do PSF na saúde dos municípios brasileiros.

Tamanha tarefa exige a institucionalização de processos estruturados e sistemáticos de avaliação dessa estratégia nas suas mais variadas dimensões como, por exemplo, atenção à saúde, gestão, impacto sobre o perfil epidemiológico e a participação social. Esta última dimensão foi relembrada por Teixeira ${ }^{(1)}$, ao citar Maria Cecília Donnangelo, Que nos ensinou em 1979, no tocante à reforma: "se fará com a população ou não se fará". Isso nos faz "aprender a aprender" Que as tecnologias aplicadas aos processos de monitoramento e avaliação devem estar entrelaçadas com o compromisso ético, humanístico e social com as famílias cuidadas pelas equipes do PSF, nos seus espaços de convivência.

Novas práticas de saúde devem ser orientadas pela essência do SUS. Há Que se fortalecer o diálogo de forma permanente, franca e democrática, com todas as forças sociais, tendo em vista um novo pacto federativo: a consolidação da política prevista na nossa Constituição. Concordamos com as idéias de Sobrinho de Que é preciso vencer as muralhas do liberalismo econômico, dos privilégios de classe, dos corporativismos Que à medida Que o sistema único de saúde avança oporão uma resistência cada vez mais encarniçada, aberta ou disfarçada. No caso do PSF, tal disfarce é potencializado pela inércia de uma agenda incompleta. Incompleta porQue não conseguiu, ao longo dos 13 anos de implantação e implementação, ampliar as condições para a superação efetiva do modelo biomédico hegemônico nas políticas públicas do setor saúde no Brasil.

\section{REFERÊNCIAS}

I. Teixeira CF. A mudança do modelo de atenção à saúde no SUS: desatando nós, criando laços. Brasil. Saúde em Debate 2003, 27(65); 257-77.

2. Arouca ASS. O dilema preventivista: contribuição para compreensão e crítica da medicina preventiva [tese]. Campinas (SP): Faculdade de Ciências Médicas na Universidade Estadual de Campinas; 1975

3. Donnangello MCF. Saúde e sociedade. São Paulo (SP): Duas Cidades; 1976.

4. Paim JS. Medicina Preventiva e Social no Brasil: modelos, crises e perspectivas. Saúde em Debate 1981; (1 1): 57-9.

5. Paim IS. A Reforma Sanitária e os modelos assistenciais In: Rouquayrol MZ. Epidemiologia \& saúde. Rio de Janeiro (RI): MEDSI; 1994. p. 455-66.

6. Paim IS. Saúde da Família. In: Saúde, política e reforma sanitária. Espaço de reflexão e de práticas contra hegemônicas? Salvador (BA): CEPS/ISC; 2002. p. 363.

7. García JC. Pensamento social em saúde na América Latina. São Paulo (SP): Cortez; 1989.

8. Mendes-Gonçalves RB. Medicina e história: as raízes sociais do trabalho médico [dissertação]. São Paulo (SP): Departamento de Medicina Preventiva, Universidade de São 
Sousa MF.

Paulo (USP); 1979.

9. Teixeira CF. Políticas de saúde no Brasil: situação atual e desafios estratégicos. Saúde em Debate 1991; (35): 4- 10.

10. Teixeira CF. Um pouco de história....reconstruindo o debate sobre modelos de atenção à Saúde no Brasil. In: Promoção e vigilância da saúde. Salvador (BA): 2002. p.1 I-22.

11. Ministério da Saúde (BR). Portaria no 3.925 de 13 de Novembro de 1998, Que aprova o Manual para Organização da Atenção Básica no Sistema Único de Saúde. Diário Oficial da União 199813 nov.
12. Lefevre F, Lefevre AMC. Depoimentos e discursos. Brasília (DF): Liberlivro Editora; 2005.

13. Ministério da Saúde (BR). Sistema de Informação da Atenção Básica - SIAB. Brasília (DF): Ministério da Saúde; 2007.

14. Sousa MF. Gestão da atenção básica: redefinindo contexto e possibilidades. Saúde em Debate 2000; (21): 7-14.

15. Sobrinho EJMA. O PSF nos grandes municípios: São Paulo, um investimento à parte! In: Sousa MF, organizador. Os sinais vermelhos do PSF. São Paulo (SP): Hucitec; 2002. p. 47-68. 